\title{
COVERING DIMENSION IN FINITE-DIMENSIONAL METRIC SPACES
}

\author{
JAPHETH HALL, JR.
}

\begin{abstract}
Let $P: 2^{V} \rightarrow 2^{V}$ be a structure in a topological space $V$ such that $P(\varnothing)=\varnothing, P(\{x\})=\{x\}$ if $x \in V$, and $P(Z)$ is closed if $Z \subseteq V$. If $G$ is a covering of $V$, let $G_{x}=\{X \in G: x \in X\}$. If $X$ is a set and $Y$ is a set, let $|X|$ denote the cardinal number of $X$ and $X-Y=$ $\{x \in X: x \notin Y\}$. Let $n$ be an integer such that $n \geqq-1$. $\operatorname{dim}_{P} V$ is defined as follows: $\operatorname{dim}_{P} V=-1$ if $V=\varnothing$. If $V \neq \varnothing$, then $\operatorname{dim}_{P} V=$ $n$ if (1) for each finite open covering $G$ of $V$, there is an open refinement $H$ of $G$ such that $\left|H_{x}\right| \leqq n+1$ if $x \in V$; and (2) there is a finite open covering $G$ of $V$ such that if $H$ is an open refinement of $G$, then $\left|H_{x}\right| \geqq n+1$ for some $x \in V$. We say that $P$ has property (*) if for each nonempty open $Y \subseteq V$ and each $X \subseteq V$ such that $P(X) \neq V$ and $x \notin P(X-\{x\})$ whenever $x \in X$ and each $x \in[V-P(X)],[Y-P(X)] \cap$ $P(X \cup\{x\}) \neq \varnothing$. THEOREM. If $V$ is a metric space, $P$ has property (*), $B \subseteq V, B$ is finite, $P(B)=V$ and $x \notin P(B-\{x\})$ if $x \in B$, then $\operatorname{dim}_{P} V=$ $|B|-1$.
\end{abstract}

1. Introduction. It is known [5, pp. 9, 93-99] that the covering dimension of each finite-dimensional Euclidean space $E^{n}$ is $n$, the usual dimension. The purpose of this paper is to present a short proof of this simply stated fact.

It is crucial that each finite-dimensional Euclidean space is a topological space $V$ in which there is a structure $P: 2^{V} \rightarrow 2^{V}[4, \mathrm{p}$. 317] such that $P$ is a closure structure having the exchange property ([2], [3], and [4]), $P(\varnothing)=\varnothing, P(\{x\})=\{x\}$ for each $x \in V$, and $P(Z)$ is closed for each $Z \subseteq V$. Indeed, if $V=E^{n}$, then the linear variety structure in $V$ will suffice as $P$, that is, if $X \subseteq V$, then $P(X)$ is the collection of all finite linear combinations of elements of $X$ with coefficients summing to 1 .

Consider a structure $P$ in a set $V$ and a subset $X$ of $V$. By definition, $X$ is $P$-independent ([2] and [3]) if $x \notin P(X-\{x\})$ for each $x \in X ; X$ is $a P$-basis of $V$ if $X$ is $P$-independent and $P(X)=V$. By definition, the $P$ dimension of $V, P$-dim $V$, exists if any two $P$-bases of $V$ have the same cardinal number. If $P$-dim $V$ exists, then $P$-dim $V$ is the cardinal number

Received by the editors November 3, 1972.

AMS (MOS) subject classifications (1970). Primary 02K20, 06A10; Secondary $54 \mathrm{C} 10$.

Key words and phrases. Covering dimension, covering dimension relative to structures in topological spaces, finite-dimensional metric spaces.

(c) American Mathematical Society 1973 
of a $P$-basis of $V$. It is known ([2] and [3]) that if $P$ is a closure structure having the exchange property and $V$ has a finite $P$-basis, then $P$-dim $V$ exists.

If $G$ is a covering of a set $V$ and $x \in V$, then the symbol $G_{x}$ shall denote $\{X \in G: x \in X\}$. If $X$ is a set and $Y$ is a set, the symbol $X-Y$ shall denote $\{x \in X: x \notin Y\}$, and the symbol $|X|$ shall denote the cardinal number of $X$. Throughout the remainder of this paper it is assumed that $V$ is a topological space and $P$ is a structure in $V$ such that $P(\varnothing)=\varnothing, P(\{x\})=\{x\}$ for each $x \in V$ and $P(Z)$ is closed for each $Z \subseteq V$.

The covering dimension of $V$ relative to $P, \operatorname{dim}_{P} V$, is defined as follows: $\operatorname{dim}_{1}, V=-1$ if $V=\varnothing$. If $V \neq \varnothing$ and $n$ is a cardinal number, then $\operatorname{dim}_{1}, V=$ $n$ if (1) and (2) are true: (1) For each finite open covering $G$ of $V$, there is an open refinement $H$ of $G$ [an open covering of $V$ such that if $X \in H$, then $X \subseteq Y$ for some $Y \in G]$ such that $\left|H_{x}\right| \leqq n+1$ for each $x \in V$, and (2) There is a finite open covering $G$ of $V$ such that if $H$ is an open refinement of $G$, then $\left|H_{x}\right| \geqq n+1$ for some $x \in V$.

We say that $P$ has property (*) if for each nonempty open subset $Y$ of $V$ and each $P$-independent subset $X$ of $V$ such that $X$ is not a $P$-basis of $V$ and each $x \in[V-P(X)], Y-P(X)$ contains an element of $P(X \cup\{x\})$.

It is shown (Theorem 1 ) that if $G$ is a finite open covering of $V$ and $B$ is a $P$-basis of $V$, then there is an open refinement $H_{B}$ of $G$ such that $\left|\left(H_{B}\right)_{x}\right| \leqq|B|$ for each $x \in V$; and (Theorem 2) that if $V$ is a metric space and $P$ has property (*) while $B$ is a finite $P$-basis of $V$, then there is a finite open covering $G_{13}$ of $V$ such that if $H$ is an open refinement of $G_{B}$, then $\left|H_{x}\right| \geqq|B|$ for some $x \in V$. It follows (Theorem 3 ) that if $V$ is a metric space and $P$ has property (*) while $B$ is a finite $P$-basis of $V$, then $\operatorname{dim}_{1}, V=|B|-1$.

2. Main results. If $V$ is a metric space, then the following notation will be used: If $r$ is a positive real number and $X$ is a nonempty subset of $V$, then the symbol $X_{r}$ shall denote $\{x \in V: d(x, X)<r\}$, where $d$ is the metric on $V$. The term "poset" $[1$, p. 1] will be used to refer to a pair $(W, R)$ such that $W$ is a set and $R$ is a partial order relation on $W$.

THEOREM 1. If $G$ is a finite open covering of $V$ and $B$ is a $P$-basis of $V$, then there is an open refinement $H_{B}$ of $G$ such that $\left|\left(H_{B}\right)_{x}\right| \leqq|B|$ for each $x \in V$.

Proof. Assume that $G$ is a finite open covering of $V$, and that $B$ is a $P$-basis of $V$. Since $P(\varnothing)=\varnothing$ and $P(\{x\})=\{x\}$ for each $x \in V$, it follows that if $B=\varnothing$ or $B=\{x\}$ for some $x \in V$, then $V=P(B)=B$, so that $G=\{V\}$. Hence, if $B=\varnothing$ or $B=\{x\}$ for some $x \in V$, then let $H_{B}=G$. Consider the case that $|B|>1$. Let $b \in B$. Using Hausdorff's maximal principle [1, 
p. 192], extend the chain $\{\varnothing\}$ of the poset $\left(2^{B-\{b\}}, \subseteq\right)$ to a maximal chain $K$ of $\left(2^{B-\{b\}}, \subseteq\right)$. Since $G$ is finite, then $\bigcap G_{x}$ is open for each $x \in V$ and each element of the poset $\left(\left\{G_{x}: x \in V\right\}, \subseteq\right)=\left\{G_{x}: x \in V\right\}$ is preceded by some minimal element of $\left\{G_{x}: x \in V\right\}$. Since $P$ is monotone and $B$ is $P$-independent, it follows that each subset of $B$ is $P$-independent. Hence, since $\varnothing \in K$ and $P(\varnothing)=\varnothing$, it follows that the collection $H$ of all $\left(\cap G_{x}\right) \cap$ $[V-P(X)]$ such that $G_{x}$ is a minimal element of $\left\{G_{y}: y \in V\right\}$ and $X \in K$ is an open refinement of $G$. Assume that $x \in V$. Choose a minimal element $G_{c}$ of $\left\{G_{y}: y \in V\right\}$ such that $G_{c} \subseteq G_{x}$. Then $\bigcap G_{x} \subseteq \bigcap G_{c}$ while $x \in \bigcap G_{x}$. It follows that the elements of $H_{x}$ are among the sets $\left(\cap G_{y}\right) \cap[V-P(X)]$ such that $X \in K$ and $G_{v}$ is a minimal element of $\left\{G_{z}: z \in V\right\}$ while $|\{V-P(X): X \in K\}|=|B|$. Therefore, $\left|H_{x}\right| \leqq|B|$. Let $H_{B}=H$. The proof is complete.

THEOREM 2. If $V$ is a metric space and $P$ has property (*) while $B$ is a finite $P$-basis of $V$, then there is a finite open covering $G_{B}$ of $V$ such that if $H$ is an open refinement of $G_{B}$, then $\left|H_{x}\right| \geqq|B|$ for some $x \in V$.

Proof. Assume that $V$ is a metric space such that $P$ has property $(*)$ while $B$ is a finite $P$-basis of $V$. If $B=\varnothing$ or $B=\{x\}$ for some $x \in V$, then let $G_{B}=\{V\}$. Consider the case that $|B|>1$. Let $n$ be a positive integer such that $|B|=n+1$. Let $B$ consist of exactly $n+1$ elements $x_{i}$ of $V$, with $1 \leqq i \leqq n+1$. Let $X_{n+1}=B$. If $0 \leqq k \leqq n$, then let $X_{n-k}=X_{(n-k)+1}-\left\{x_{n-k}\right\}$. Let $r$ be a positive real number. Let $G$ consist of precisely the following sets: $P\left(X_{k}\right)_{r}$, with $1 \leqq k \leqq n$ and $X_{0}=\varnothing$. Since $P(Z)$ is closed for each $Z \subseteq V$, it follows that $G$ is a finite collection of open subsets of $V$. Consider any element $x$ of $V$. If $x \in P\left(X_{1}\right)$, then $x \in\left[P\left(X_{1}\right)_{r}-P\left(X_{k-1}\right)\right] \subseteq \bigcup G$. If $x \notin P\left(X_{1}\right)$, let $m$ be the largest positive integer such that $x \in P\left(X_{m}\right)$, so that $x \in\left[P\left(X_{m+1}\right)_{r}-P\left(X_{m}\right)\right] \subseteq \bigcup G$. It follows that $G$ is a finite open covering of $V$. Assume that $H$ is an open refinement of $G$. If $1 \leqq k \leqq n+1$, let $H_{k}$ be the collection of all $X \in H$ such that $x \in X$ for some $x \in\left[P\left(X_{k}\right)-\right.$ $\left.P\left(X_{k-1}\right)\right]$. Since $x_{1} \in\left[P\left(X_{1}\right)-P\left(X_{0}\right)\right]$, then $H_{1}$ contains an element $Y_{1}$. It follows from $(*)$ that $Y_{1}-P\left(X_{1}\right)$ contains an element $y_{1}$ of $P\left(X_{2}\right)$, so that $y_{1} \in\left[P\left(X_{2}\right)-P\left(X_{1}\right)\right]$. Hence $y_{1} \in Y_{2}$ for some $Y_{2} \in H_{2}$ such that $Y_{2} \notin H_{1}$. If $n \geqq 2$, then it follows from (*) that $\left(Y_{1} \cap Y_{2}\right)-P\left(X_{2}\right)$ contains an element $y_{2}$ of $P\left(X_{3}\right)$, so that $y_{2} \in\left[P\left(X_{3}\right)-P\left(X_{2}\right)\right]$ and $Y_{2} \notin\left[P\left(X_{i}\right)-P\left(X_{i-1}\right)\right]$ if $1 \leqq i \leqq 2$. It follows by induction that there is a collection $\left\{Y_{i}: 1 \leqq i \leqq n\right\}$ of elements of $H$ such that if $1 \leqq k \leqq n$, then $\left(\bigcap\left\{Y_{i}: 1 \leqq i \leqq k\right\}\right)-P\left(X_{k}\right)$ contains an element $y_{k}$ of $P\left(X_{k+1}\right)$ while $Y_{i} \notin H_{j}$ if $1 \leqq j<i \leqq k$. It follows that $y_{n} \in\left[P\left(X_{n+1}\right)-P\left(X_{n}\right)\right]$ and $y_{n} \notin\left[P\left(X_{i}\right)-P\left(X_{i-1}\right)\right]$ if $1 \leqq i \leqq n$. Let $x=y_{n}$. Then $x \in Y_{n+1}$ for some $Y_{n+1} \in H_{n+1}$ such that $Y_{n+1} \notin H_{i}$ if $1 \leqq i \leqq n$. It follows that $\left\{Y_{i}: 1 \leqq i \leqq n+1\right\} \subseteq H_{x}$, so that $\left|H_{x}\right| \geqq n+1$. Therefore, $\left|H_{y}\right| \geqq|B|$ for some $y \in V$. The proof is complete. 
THEOREM 3. If $V$ is a metric space and $P$ has property (*) while $B$ is a finite $P$-basis of $V$, then $\operatorname{dim}_{P} V=|B|-1$.

Proof. Suppose that $V$ is a metric space such that $P$ has property (*) while $B$ is a finite $P$-basis of $V$. It follows from Theorem 1 that if $G$ is a finite open covering of $V$, then there is an open refinement $H_{B}$ of $G$ such that $\left|\left(H_{B}\right)_{x}\right| \leqq|B|$ for each $x \in V$. Application of Theorem 2 yields a finite open covering $G_{B}$ of $V$ such that if $H$ is an open refinement of $G_{B}$, then $\left|H_{x}\right| \geqq|B|$ for some $x \in V$. Therefore, $\operatorname{dim}_{P} V=|B|-1$. The proof is complete.

COROLlary. If $V$ is a metric space and $P$ has property (*) while $V$ has a finite $P$-basis and $P$-dim $V$ exists, then $\operatorname{dim}_{P} V=[P-\operatorname{dim} V]-1$.

The linear variety structure $Q$ in $E^{n}$ is a closure structure having the exchange property and property (*), $Q$-dim $E^{n}$ exists and $E^{n}$ has a finite $Q$-basis of exactly $n+1$ elements. Therefore, $\operatorname{dim}_{Q} E^{n}=n$.

\section{REFERENCES}

1. G. Birkhoff, Lattice theory, 3rd ed., Amer. Math. Soc. Colloq. Publ., vol. 25, Amer. Math. Soc., Providence, R.I., 1967. MR 37 \#2638.

2. M. N. Bleicher and E. Marczewski, Remarks on dependence relations and closure operators, Colloq. Math. 9 (1962), 209-212. MR 26 \#58.

3. M. N. Bleicher and G. B. Preston, Abstract linear dependence relations, Publ. Math. Debrecen 8 (1961), 55-63. MR 24 \#A124.

4. J. Hall, Jr., The independence of certain axioms of structures in sets, Proc. Amer. Math. Soc. 31 (1972), 317-325. MR 45 \#141.

5. J. Nagata, Modern dimension theory, Bibliotheca Math., vol. 6, Interscience, New York, 1965. MR 34 \#8380.

Department of Mathematics, Stillman College, Tuscaloosa, Alabama 35401 